\title{
Praying that this was the last state of emergency
}

\author{
Yoshiki Hirooka $^{1}$
}

Received: 1 October 2021 / Accepted: 6 October 2021

(c) The Author(s), under exclusive licence to The Japan Society of Ultrasonics in Medicine 2021

This issue of the Journal of Medical Ultrasonics (JMU) is scheduled to be released on October 15, but I am writing this Editor's Note on October 1. The state of emergency was lifted on September 30 in the areas of Japan for which a state of emergency had been declared, resulting in the relaxation of various restrictions starting today. Japan's ruling Liberal Democratic Party elected a new leader on September 29. The terms of office of members of the House of Representatives are set to expire on October 21, and I anticipate that we will see a number of developments in the political world in the run-up to the general election. It states the following on the website of Japan's Ministry of Internal Affairs and Communications: "A general election is an election held to select all members of the House of Representatives. Singleseat constituency elections and proportional representation elections are held on the same polling day. There are two types of general elections: one held when the terms of office (4 years) of members of the House of Representatives expire, and one held when the House of Representatives is dissolved." It is not known at the present time which of the two types of general election will be held this time around, but I think there is no doubt that laying out how COVID-19 and the various changes resulting from it will shake out after COVID-19 ends (although defining "end" is difficult) will be a major issue in the general election. And before I knew it, the 2020 Tokyo Olympic/Paralympic Games ended. I think that examination of what impact this had on Japan may be another issue in the election. I have a feeling that we will be moving forward while bracing ourselves for a restless world and a future that will remain uncertain for some time. In any event, I think one thing is certain: all we can do is do the best we can going forward in the situation we find ourselves in. At JMU, as well, the entire Editorial Board (members/ secretariat) will devote itself to providing our readers with

Yoshiki Hirooka

hirooka@med.nagoya-u.ac.jp

1 Department of Gastroenterology and Hepatology, Fujita Health University, Toyoake, Aichi, Japan articles that they will find informative. Thank you for your continued support.

Moving on, the contents of this issue are more meaty than ever with over 30 articles. Of particular note are the following three features included in this issue:

1. Recent progress in basic research useful for the interpretation of ultrasound diagnostic images (project lead: Naotaka Nitta).

2. Quantitative assessment of liver steatosis using ultrasound (project lead: Katsutoshi Sugimoto).

3. Role of ultrasonography in the evaluation of autoimmune pancreatitis (project lead: Eizaburo Ohno).

JMU is one of the few academic journals in the world that covers both clinical and basic research related to ultrasound. In the feature from Dr. Nitta, the basics of ultrasound that every clinician who actually uses it should know are explained in a manner that is very easy to understand. The feature from Dr. Sugimoto consists of a detailed review of the role of not only ultrasound imaging but also computed tomography (CT), magnetic resonance imaging (MRI), and histopathology in the assessment of liver steatosis, which is currently one of the pillars of hepatic disease research. The feature from Dr. Ohno covers ultrasound imaging, endoscopy findings, and CT/MRI findings, as well as the histopathology that underlies these, as they relate to autoimmune pancreatitis, which was first advocated in Japan, and like the feature on liver steatosis, all necessary autoimmune pancreatitis-related knowledge can be acquired by reading this feature. We are confident that these features alone cover everything related to liver steatosis and autoimmune pancreatitis, and that our readers will be able to acquire the foundation to pursue new research just by reading these features. The Editorial Board has faith that these features will result in many research presentations and the publication of many articles.

The Editorial Board has come up with a variety of strategies over the years to raise JMU's impact factor and its international profile. One such strategy has been to publish 
features that appeal to our readers in disciplines that deal with ultrasound. JMU is a journal that publishes papers on basic and clinical medicine related to ultrasound, but features are put together in a way that makes them appealing, such as allowing the inclusion of CT or MRI reviews and pathology reviews like in this issue, so that readers can grasp the full picture of a condition by becoming acquainted with the relationship between ultrasound and other modalities or histopathology. We publish features that allow an author writing a paper in a certain domain to get all the information they need by simply reading JMU's feature. We publish features that provide an understanding of the full picture of a domain by simply using the feature, and that cover all of the necessary references for those carrying out research and those writing papers on ultrasound not only in clinical medicine domains but also in basic medicine domains.
JMU's Editorial Board is comprised of a variety of experts in domains such as basic engineering and clinical medicine. We plan to continue to publish many features that will benefit our readers, covering not only a wide range of the most up-to-date topics but also topics that will provide an understanding of the basics. The Editorial Board hopes to mobilize all of its energy to produce a JMU that receives wide recognition from researchers from all over the world involved in medical ultrasonics.

Publisher's Note Springer Nature remains neutral with regard to jurisdictional claims in published maps and institutional affiliations. 